\title{
LA METODOLOGÍA Q: \\ MÁS QUE UNA TÉCNICA DE INVESTIGACIÓN*
}

Nilson Genaro Valencia Vallejo**

\begin{abstract}
In this article introduce a research technique named $Q$ Methodology, based on my own experience during my research internship with the TECNICE group, encouraged and supported by COLCIENCIAS and Universidad Pedagógica Nacional through the "Jovenes Investigadores" Program. The field of study of this technique is the human subjectivity and I introduce an application case to the learning of complex conceptual systems using a hypertext environment with a software agent programmed according to such methodology This application becomes an innovative projection for learning development in several knowledge domains and design of computer based learning environments.
\end{abstract}

\section{RESUMEN}

En este artículo se presenta una técnica de investigación denominada Metodología $Q$, a partir de la experiencia vivida por el autor del presente artículo, durante su pasantía como investigador e integrante del grupo TECNICE, en calidad de becario en el programa Jóvenes investigadores, impulsado y financiado por Colciencias y la Universidad Pedagógica Nacional. Se ubica este desarrollo en el contexto de la investigación sobre la subjetividad humana y se presenta una experiencia de aplicación a la formación de sistemas conceptuales complejos, combinando ambientes hipertoxtuales con un agente de software programado siguiendo la lógica de la metodología en mención. Esta aplicación constituye una proyección novedosa al desarrollo del aprendizaje en las diferentes áreas del conocimiento y al diseño de ambientes de aprendizaje basados en computador.

Palabras clave: Metodología $Q$, técnica $Q$, investigación, subjetividad, estudio del comportamiento, software, agente $Q$,

\footnotetext{
* Trabajo orientado por Luis Facundo Maldonado Granados, Ph.D., profesor de la Universidad Pedagógica Nacional de Bogotá.

-- Candidato a Magíster en Tecnologías de la Información Aplicadas a u Educación, Universidad Pedagógica Nacional.
} 


\section{Introducción: una vivencia compartida}

Jóvenes investigadores de Colciencias es uno los pocos programas que existen en el país, con el fin de contribuir en la formación de los jóvenes talentos nacionales, en las diferentes áreas de investigación en ciencia y tecnología, mediante la experiencia de participación directa en la práctica investigativa. Ha vinculado, a la fecha, a más de 500 jóvenes en todo el país, con el propósito de formarlos y apoyarlos para que puedan colaborar en las diferentes actividades que se desarrollan en el interior de los centros y grupos de investigación, tanto en universidades públicas como privadas. La vinculación de estos jóvenes es de tiempo completo, durante un período no superior a doce meses.

En el mes de marzo de 2002, el autor se vincula, mediante el programa en mención, al grupo TECNICE, dirigido por el doctor Luis Facundo Maldonado Granados. El objetivo principal de investigación de este grupo, es el uso de programas de computador orientados a comprender y desarrollar la autonomía en el aprendizaje humano. La evolución conceptual del grupo le ha llevado a integrar desarrollos que tienen como dominio de conocimiento, principalmente, el razonamiento espacial en el contexto del diseño y de áreas de la educación básica y vocacional como la matemática, el español, las ciencias de la naturaleza y las ciencias sociales. Estos programas se orientan al desarrollo de dimensiones del aprendizaje significativo y de la colaboración.

Actualmente, las diversas líneas de investigación que lidera el grupo se constituyen apoyo estructural de los programas de formación a nivel de especialización y maestría en "Tecnologías de la Información Aplicadas a la Educación" y de Programas de Formación Permanente de Docentes en la Gestión de Proyectos Educativos en Informática y Diseño de ambientes computacionales o Software Educativo de la Universidad Pedagógica Nacional.

Para el autor, lograr su vinculación al grupo TECNICE constituye una gran satisfacción, por el compromiso y liderazgo que caracteriza al grupo. Ha sido un año de arduo trabajo y múltiples esfuerzos por sacar adelante los compromisos adquiridos, pero, a la vez, de muchos logros, tanto a nivel nacional como internacional, debido a su fortaleza, reconocimiento y acreditación. Dentro de las diversas actividades que se desarrollaron, se pueden destacar: la coordinación de La Red Iberoamericana de Informática Educativa -Capítulo Colombia - RIBIECOL, que forma parte del Subprograma VII de Electrónica e Informática Aplicadas del CYTED y se encuentra constituida por instituciones y grupos que desarrollan o aplican tecnologías de la información a la solución de problemas educativos; la organización del "IV Foro Colombiano de Informática Educativa", que tuvo lugar en la ciudad de Cartagena en el mes de abril; el "VI Congreso y VI Premio Colombiano de Informática Educativa", realizado en Medellín en el mes de julio yen el mes de noviembre; el Encuentro de Práctica Docente y Tecnologías de la Información "Los jóvenes construimos el futuro de nuestra profesión", evento realizado en la Biblioteca Virgilio Barco, ubicada en la ciudad de Bogotá; el desarrollo de dos proyectos de investigación - el primero financiado por Colciencias, titulado: "Agentes Inteligentes de Software Orientados al Juego de Roles para el Aprendizaje Significativo de la Constitución Colombiana" y el segundo, financiado por el Centro de Investigaciones de la Universidad Pedagógica Nacional, CIUP, titulado: "Agente $Q$ para la Estructuración de Sistemas Conceptuales: validación en entornos hipertextuales y textuales".

El primer proyecto busca establecer la relación entre los niveles de argumentación logrados por los sujetos que juegan uno, dos o tres roles diferentes en la solución de problemas representados en ambientes computacionales, dentro del marco de la 
Constitución Nacional, frente a aquellos que, para su análisis, se basan en el estudio de casos sin la utilización del software. El segundo, está centrado en el diseño y validación de un software para manejo, registro y gradación de estructuras conceptuales con base en la metodología $Q$ e incorpora la tecnología informática a la formación de competencias cognitivas, presentando alternativas para el estudio y organización de información disponible en formato hipertextual o en documentos impresos. Esto artículo se centra en la experiencia vivida con el segundo proyecto.

\section{Perspectivas históricas de la metodología $\mathbf{Q}$}

La metodología Q, se presentó en Carta a la Naturaleza, escrita por William Stephenson, en 1935, físico (Ph.D. 1926, Universidad de Durham) y psicólogo (Ph.D. 1929, Universidad de Londres). A menudo, se le asocia con el análisis cuantitativo debido a que hace tratamiento de datos con base en análisis factorial. La metodología $Q$ "combina elementos, tanto de la investigación cualitativa, como cuantitativa y en algunos casos establece un puente entre las dos (Venda \& Castaño, 1984).

El propósito de Stephenson (1935), al introducir la metodología Q en su investigación, es avanzar en el entendimiento de la conducta humana. Esta metodología fue apartada de su propósito inicial, a causa de una gran controversia por parte de la Academia de Psicología, la cual pretendía que su implementación se llevará a cabo más en las áreas de la salud, en lo alusivo al estudio o exploración de la subjetividad humana. No obstante, ha sido notable su aplicación en el campo de las comunicaciones y de las ciencias políticas, y recientemente en las ciencias de la salud; en el campo de la Psicología aún se utiliza muy poco. Recientemente, una generación de psicólogos jóvenes está redescubriendo la metodología $Q$, en concordancia con la visión que William Stephenson promovió por más de medio siglo.

Las referencias muestran que Stephenson (1935) estaba interesado en develar la subjetividad involucrada en situaciones a las cuales se enfrenta el individuo en su cotidianidad, en el medio social con el cual interactúa; por ejemplo, en los juicios estéticos, la interpretación poética, las actitudes políticas, las apreciaciones de cuidado de salud, las experiencias de desamparo, las perspectivas en la vida y el cosmos, entre otros. Es la vida vivida desde el punto de vista de quien la vive, lo cual generalmente supera la capacidad expresiva de los procedimientos cuantitativos; es, en este sentido, como la metodología $Q$ se diseñó para examinar la subjetividad humana y llamar la atención del investigador cualitativo.

\section{¿Qué es la metodología Q?}

Para Stephenson (1953), la metodología Q es el nombre genérico utilizado para caracterizar un conjunto de ideas filosóficas, psicológicas, pedagógicas, estadísticas y psicométricas, orientadas a la investigación de la subjetividad humana. Mckewon y Thomas (1988), también la definen como el estudio de la subjetividad o punto de vista de una persona en cualquier materia de importancia personal o social (1).

El método $Q$ revela ordenamientos por rango de la opinión de las personas. Cada ordenamiento se denomina también factor, y es considerado individualmente o en grupo.

El factor individual se define como el conjunto de opiniones o posiciones subjetivas adoptadas por cada individuo en particular y de acuerdo con su propio punto de vista. El método $Q$ enfatiza la preservación de puntos de vista individuales; de hecho, Stephenson 
(1953) insistió en la selección de personas conocidas por tener intereses particulares. El método normalmente emplea un número pequeño de sujetos que puedan responder la prueba. El método $Q$ no se diseña para determinar cuántas personas en el mundo muestran una tendencia, Brown (1980) afirma que su meta es dar estructura a las opiniones subjetivas que en ellos se pueden observar y estudiar (2).

Consecuentemente, el factor considerado como grupo, es concebido como un compuesto formado por los puntos de vista de la gente que piensa el asunto de una manera particular. Los factores $Q$ son grupos de "gustos" de las personas que están unidas o identificadas por creencias comunes, actitudes y opiniones, Singer (1997). El método $Q$ revela el punto de vista común de opiniones a través del proceso matemático de análisis de factores. Cada factor realmente es un compuesto o un grupo de las personas que piensan sobre el tema de una manera similar. La metodología $Q$ se implementa mediante una serie de procedimientos llamados Técnica 0, (3).

\section{La técnica $\mathbf{Q}$}

La técnica $Q$ se centra particularmente en la clasificación de grupos de tarjetas denominadas clasificaciones $Q$ y en las correlaciones entre las respuestas 0 clasificaciones realizadas por diferentes individuos. Los ordenamientos realizados por cada persona en particular, son tratados individualmente y las clasificaciones consideradas grupalmente son tratadas como unidades; es decir, cada grupo conforma una unidad para efectos de interreación entre grupos.

La técnica $Q$ es sobre todo una forma compleja de ordenar por rangos las tarjetas que contienen los reactivos (estímulos, proposiciones, palabras individuales, frases, fotografías, composiciones musicales, etc.) sobre un asunto de interés, y después asignarles valores numéricos a los subconjuntos de tales tarjetas con propósitos estadísticos, Kerlinger (1997). Un acercamiento de la metodología Q preguntaría a un grupo de individuos cómo se sienten con respecto a las propuestas políticas de cuatro sujetos que son candidatos al cargo de presidente en un país y las organizaran en orden de más acuerdo a menos acuerdo (4).

Todos los ordenamientos realizados por el grupo son subjetivos; es decir, no habría ninguna respuesta correcta o incorrecta. Algunas personas se sentirían identificadas libremente por una de las propuestas políticas presentadas, otros pueden tener influencia por alguna propuesta a raíz de nexos familiares, sentir-se miembros de un partido político o citar que están envueltos en asociaciones de participación política en busca de un bien particular. La variación en la clasificación de los ordenamientos se sujetaría al análisis estadístico para revelar modelos dominantes de creencia. De esta manera, el método $Q$ identificaría cómo los individuos perciben una situación dada.

\section{Trabajos píoneros}

La metodología $Q$ ha sido utilizada de manera continuada en el análisis de sistemas conceptuales y de valores en varias disciplinas (Stephenson, 1953). Generalmente, se relaciona con los métodos estadísticos de estudio del comportamiento de una manera rigurosa y natural; sin embargo, su base está en las valoraciones cualitativas que hacen las personas de conjuntos de expresiones conceptuales. Por sus aplicaciones a diferentes formas de valoración, como el arte, la política y la ética, ha tenido mucho interés para las investigaciones de enfoque cualitativo. 
Sontag (1968) realizó un estudio $Q$ de la relación entre las actitudes hacia la educación y hacia las percepciones de los comportamientos deseables de enseñanza, para lo cual usó una clasificación $\mathrm{O}$ de 80 reactivos que eran breves descripciones de una gran variedad de comportamientos de enseñanza (Quintero, 2002, pp. 10-12) (5).

Así mismo, la metodología $Q$ fue utilizada para estudiar las relaciones económicas entre China y Estados Unidos, a partir de los últimos desarrollos comerciales; el trabajo consistió en que grupos de individuos de las dos naciones construyeran sus propias historias y a través de ellas se revelaran las diferencias y similitudes que en materia comercial caracterizaba a las dos culturas.

Lasweel Harol llevó a cabo un estudio con metodología Q, en Sudáfrica, sobre la experiencia representativa y la clarificación de valores, con el fin de ver el rol que juegan en la clasificación de metas en los procesos políticos; en este estudio se incorporó la técnica $Q$ para proveer bases operacionales con el propósito de develar qué resultados sobre juicios valorativos habían surgido en los individuos, después de la ley del Apartheid sobre discriminación racial (5).

En la Universidad de Harvard, se llevó a cabo una investigación acerca de la concepción de privacidad reflejada en la normatividad o reglamento del alma mater. Los resultados acerca de la privacidad que constituyen el propósito de este estudio, se realizaron con base en el supuesto difundido de la existencia de áreas de creencias individuales y actividades personales que no conciernen al gobierno, grupos $u$ otras personas ajenas a dicha institución (Quintero, 2002, pp. 10-12) (5).

En 1996, en La federación rusa, se investigó sobre el mercadeo de la sociedad consumidora rusa con el propósito de involucrar parte del proceso de introducir técnicas de investigación modernas en el marketing para superar la rudimentaria investigación que predominaba en el momento (5).

Quintero (2002) desarrolla una investigación que tiene como objetivo mostrar la aplicación de la metodología $Q$ en el estudio de la orientación interna o externa en estudiantes de bachillerato. Se basé en la construcción de cuarenta y ocho proposiciones verbales sobre locus de control -interno o externo- y sobre los derechos a la igualdad y a un ambiente sano. Las proposiciones se presentan al sujeto a través de un prototipo de software desarrollado para adelantar la investigación, cuyo propósito fue el de identificar si las decisiones que toma cada individuo o cada grupo de individuos en los ordenamientos por rango, cambian a medida que los individuos realizan nuevos ordenamientos individuales o a medida que los grupos acuerdan o negocian nuevas clasificaciones. La experiencia se realizó en un colegio oficial en la ciudad de Bogotá con estudiantes de sexto y décimo grado (5).

\section{Una experiencia en el grupo TECNICE}

En la actualidad, el grupo de investigación TECNICE desarrolla una experiencia en la Universidad Pedagógica Nacional, donde se incorpora la metodología $Q$ mediante un programa de computador denominado Agente $Q$ orientado a favorecer el proceso de aprendizaje, con el fin de descubrir en qué grado este sistema incide en la comprensión y formación de competencias cognitivas en el estudio de textos o hipertextos.

En la Metodología $Q$, básicamente nos ubicamos ante dos ítems, en el sentido de expresiones conceptuales, la de ser un nombre o, como forma más elaborada, una 
proposición. Una expresión unitaria (no un discurso) o una proposición es un concepto. Si tenemos dos proposiciones: una a la derecha y otra a la izquierda en una pantalla — dos ítems preposicionales-, cada uno forma una unidad conceptual.

La valoración ocurre cuando tomamos una decisión: "Te vas para la izquierda o, te vas para la derecha" o cuando nos dan las dos proposiciones y nos preguntan: "¿con cuál de las dos proposiciones estás más de acuerdo?". Al responder, señalamos una de las dos y establecemos una jerarquía valorativa, la cual es el punte de interés de esta investigación.

El trabajo de TECNICE hace énfasis en los sistemas conceptuales complejos; es decir, los que están compuestos por dos o más proposiciones. Lo que hacemos con la metodología $Q$ es trabajar con conjuntos de muchas proposiciones, "pilas de proposiciones", tan grandes como se quiera (pueden ser 4, como pueden ser 100, 200, etc.). Lo que se hace es hacer compatible el concepto de memoria de capacidad limitada con el de sistema complejo de conceptos.

Cuando se habla de sistemas complejos, se toman como componentes o elementos del sistema una cantidad de proposiciones que son unitariamente conceptos. El número $\mathrm{n}$ de conceptos expresados en $n$ proposiciones son los elementos de lo que podemos llamar un sistema conceptual complejo. Entre ellos se establecen relaciones para que se constituya el sistema. La metodología $Q$ permite que el usuario construya y reconstruya el sistema conceptual complejo y que, al hacerlo, construya su propio conocimiento que conserva en memoria de largo plazo.

Tenemos una proposición independiente, luego viene otra proposición independiente y el sistema le pregunta al usuario: “¿Cuál de esas alternativas es la correcta: a o b?”. Cada vez aparece una pareja, y el sujeto establece una relación entre sus elementos. Esta decisión hace que los elementos sean ordenados en dos conjuntos. Luego, cada uno de los subconjuntos es, a su vez, ordenado mediante sucesión de decisiones similares. Esta forma de ordenamiento se ajusta a las condiciones de la memoria de trabajo del ser humano que tiene una capacidad de almacenamiento limitado —máximo 7 ítems-.

De manera sustantiva, nuestro propósito se centra en estudiar los sistemas conceptuales complejos y ver cómo se forman, e indagar si con dispositivos informáticos podemos apoyar la estructuración de sistemas conceptuales, a partir de la concepción sobre metodología Q. Este tipo de software lo podemos ubicar en la categoría de 'instrumento cognitivo', o software con el cual aprender, por contraposición al software del cual se aprende ${ }^{1}$.

A continuación se plantea la ruta metodológica desarrollada en el proyecto para llegar a validar las hipótesis planteadas.

En primera instancia, se seleccioné un dominio de conocimiento específico, constituido por tres unidades temáticas, las cuales están representadas en hipertextos desarrollados previamente por el grupo de investigación. Los hipertextos seleccionados fueron: Geografía económica de Colombia, Arte geométrico en la versión de Omar Rayo, e Historia de la grúa. La característica principal de estos hipertextos la constituye el hecho

\footnotetext{
${ }^{1}$ Seminario de investigación realizado por el grupo TECNICE, Bogotá, mayo de 2002. 
de que sus dominios de conocimiento están representados mediante sistemas de marcos ${ }^{2}$.

Posteriormente se elaboró una prueba de competencias, tomando como referencia los dominios de conocimiento seleccionados (geografía económica, arte geométrico y tecnología). Las preguntas de esta prueba se elaboraron teniendo en cuenta tres niveles de profundidad de las inferencias, los cuales están determinados por el número de niveles involucrados en el sistema de marcos. Para su aplicación, se diseñé un programa de computador que evalúa el desempeño del estudiante en cada una de las temáticas.

El Agente $Q$ es un programa de computador que se adicioné a cada hipertexto. Este agente identifica la estructura jerárquica del hipertexto y la representa en un sistema de tarjetas, con las cuales puede jugar el usuario. El objetivo del estudiante es organizar las tarjetas que el programa le presenta, de tal manera que emule el ordenamiento realizado por el experto que elaboré el hipertexto.

El ordenamiento realizado por el estudiante es valorado por el programa, que realiza un cálculo en escala decimal de 0 a 10, indicando la correlación existente entre el ordenamiento ideal —el del experto - y el realizado por el estudiante.

Para iniciar la etapa experimental, se desarrolló un pilotaje en el Colegio Cafam de Bogotá, con estudiantes de grado noveno. Se organizaren cuatro grupos mediante asignación aleatoria de los sujetos. Un grupo estudiaba un documento impreso y luego era evaluado; otro estudiaba el mismo material usando el Agente $Q$ y era evaluado; el tercero estudiaba un hipertexto que contenía el documento impreso del primer grupo y luego era evaluado y, finalmente, el cuarte grupo estudiaba el hipertexto con Agente $Q$ y era evaluado. En estas condiciones, se desarrollé entre los sujetos lo que técnicamente se denomina "efecto de halo": Los sujetos que no usaban computador se sintieron en condiciones de inferioridad y los que tenían acceso a los programas, en condiciones de privilegio. La motivación, en consecuencia, se constituyó en preocupación para los investigadores. El diseño perdió validez, lo que obligó a hacer los ajustes correspondientes y declarar esta experiencia como experiencia piloto. Las diferencias de resultados eran atribuibles al efecto de hale o al uso del Agente Q. La doble posibilidad de explicación de los resultados se traduce en pérdida de validez del diseño.

Entonces, se propuso dividir la validación de la investigación en des etapas. La primera fue experimentar con la condición de hipertexto con Agente $Q$ y contrastarla con la condición documente escrito con Agente $Q$ con el objetivo de validar la primera hipótesis formulada en el proyecte: "Comparadas las dos formas de uso del Agente $Q$, se espera que los estudiantes que le utilizan en el estudie de un hipertexto obtengan mejores resultados que quienes lo utilizan para estudiar un documento impreso".

En la segunda etapa, se contrastaría la condición de hipertexto sin Agente $Q$ con la de Hipertexto con Agente $Q$, con el cual se busca validar la segunda hipótesis: "Tomando como variable dependiente una prueba de competencias en comprensión de textos, se esperaría que los estudiantes que usan el Agente Q, obtengan puntajes más altos".

La experimentación, en su primera etapa, se realizó en el Colegio Santa Isabel de Hungría, de Bogotá, y se estructuré de la siguiente manera: se dividió una población de 80

\footnotetext{
${ }^{2}$ Un sistema de marcos se define como una red de nodos y relaciones organizadas en una jerarquía, donde los nodos más altos representan conceptos generales y los más balos instancias específicas de esos conceptos, Sanabria (2003).
} 
estudiantes de grado noveno en des grupos; el grupo A representó la condición de hipertexto y Agente $Q$ y el grupo $B$ a la condición de texto impreso y Agente $Q$. Los estudiantes fueron asignados aleatoriamente a cada una de las dos condiciones.

A los estudiantes del grupo A se les presentó Los tres hipertextos con Agente $Q$ (uno por sesión), y realizaron la evaluación de competencias correspondiente a cada hipertexto. El grupo $B$, de igual manera que el grupo $A$, realizó el proceso explicado anteriormente, con la diferencia de que a este grupo no se le presentó el hipertexto en un ambiente computacional, sino en texto impreso.

Al terminar las sesiones de trabaje, se recogieron los resultados de los dos grupos y se realizó el tratamiento de datos utilizando un programa de software Statistics (Copyright StatSoft, Inc, 1984-2003), para hacer el análisis de varianza correspondiente, sobre los resultados de la prueba. A partir de los datos obtenidos, se confirma la hipótesis nula de la investigación. Los resultados obtenidos por los dos grupos son estadísticamente iguales. Esto significa que el Agente $Q$ como activador de proceses cognitivos, genera resultados muy similares tanto en ambientes de hipertexto como en ambientes donde se tema como base el texto impreso.

En la actualidad, realizamos la segunda etapa de la experimentación, que se desarrolla en el Colegio Distrital Juan del Corral, en la jornada de la mañana, y nos encontramos a la expectativa de los resultados, que seguramente serán publicados en un futuro inmediato.

\section{Algunas conclusiones}

En este breve artículo he tratado de dar a conocer a los lectores una aproximación general de la metodología $Q$, sin entrar en detalles técnicos. Quisiera resaltar algunas ideas suscitadas a lo largo de mi pasantía de investigación:

La primera, que la investigación es una empresa científica que exige mucho estudie y una prolongada experiencia. Hago referencia al tipo de investigación con la cual estuve en contacto durante este tiempo.

La segunda, que, si bien se están evidenciando progresos en lo que atañe a los proceses de comprensión básica en el desarrolle de la investigación social en el país, muchas de las dificultades con las que tropiezan los investigadores preceden, pura y exclusivamente, de la magnitud del estudie necesario y la estrategia metodológica para obtener resultados definitivos.

De igual manera, destace el potencial oculto existente en las nuevas técnicas de investigación, como la metodología $Q$, que fue de mi interés, en primera instancia, perlas implicaciones que ésta puede llegara tener en el campe educativo $y$, en segunda instancia, por los niveles de abstracción que puede alcanzar un aprendiz en un dominio de conocimiento especifico, tras su incorporación.

Por último, con respecte a la experiencia vivida como joven investigador, por efectos de la conclusión de la pasantía de investigación financiada por Colciencias y La Universidad Pedagógica Nacional, quiero agradecer a estas instituciones por la valiosa oportunidad brindada, la financiación asignada y por el gran aporte a nivel personal y profesional. 
También, agradezco, muy especialmente, al profesor Luis Facundo Maldonado Granados por su significativa y valiosa tutoría, al grupo de investigación TECNICE, por haberme acogido como miembro activo del mismo en sus actividades y, finalmente, al Centro de Informática de la Universidad Pedagógica Nacional.

\section{Bibliografía}

1 Brown, Steven R. Politlcal Subjectivity. New Haven, CT: Yale University Press. http ://facstaff. uww.edu/cottlec/Qarchive/ qindex.htm., 1980.

2. Brown, S. Q Methodology and Quantum Theory: Analogies and Realities Kent State University, http://facstaff.uww.edu/cottlec/Qarchive/qindex.htm., 1980.

3. Brown, S. Q Methodology and Qualitative Research for the Human Sciences. www.rz.unibw-muenchen.de/--p41 hsmk/qmethod, 1992.

4. Kerlinger, Fred N. Metodología Q. Investigación del comportamiento, (577-595). McGRAW-HILL. Tercera edición, 1996.

5. Quintero Suárez, Víctor J. Metodología Q como identificador de estructuras actitudinales que fundamentan las decisiones subjetivas. Universidad Pedagógica Nacional, 2002.

6 Maldonado Granados, Luis F. Competencias docentes en el contexto de los ambientes virtuales de aprendizaje. www.icfes.gov.co/es/fomento/gcfem/docs/ferdoce/com_cat_ins/catANC/mem2002/Pone nciaLUISFACUNDOMALDONADO. rtf., 2002.

7. Sanabria Rodríguez, Luis B. Formulemos sistemas expertos. Bogotá, Universidad Pedagógica Nacional, 2003.

8. Z., Amin. "Q methodology. A Journey into the Subjectivity of Human Mind.", en: Singapore Med J, vol. 41(8):410-414, 2002. 